\title{
An Expert System for Wind Shear Avoidance
}

\author{
Robert F. Stengel1 and D. Alexander Stratton ${ }^{2}$ \\ Princeton University \\ Department of Mechanical and Aerospace Engineering \\ Princeton, NJ
}

\begin{abstract}
A study of intelligent guidance and control concepts for protecting against the adverse effects of wind shear during aircraft takeoffs and landings is being conducted, with current emphasis on developing an expert system for wind shear avoidance. Principal objectives are to develop methods for assessing the likelihood of wind shear encounter (based on real-time information in the cockpit), for deciding what flight path to pursue (e.g., takeoff abort, landing go-around, or normal climbout or glide slope), and for using the aircraft's full potential for combating wind shear. This study requires the definition of both deterministic and statistical techniques for fusing internal and external information, for making "go/no-go"decisions, and for generating commands to the aircraft's autopilot and flight directors for both automatic and manually controlled flight. The program has begun with the development of the WindShear Safety Advisor, an expert system for pilot aiding that is based on the FAA Windshear Training Aid; a two-volume manual that presents an overview, pilot guide, training program, and substantiating data provides guidelines for this initial development. The WindShear Safety Advisor expert system currently contains over 200 rules and is coded in the LISP programming language.
\end{abstract}

\section{INTRODUCTION}

Flight in strong wind shears, especially microbursts, poses a unique and severe hazard to aircraft. The disturbance caused by the wind field may literally exceed the performance characteristics of the aircraft, making safe transit impossible even with optimal guidance and control strategies. An unusual degree of piloting skill may be required to successfully elude danger. Nevertheless, planes fly in moderate wind shear all the time; pilots learn to handle crosswinds, gustiness, and moderate frontal activity. The problem is that microbursts are random, rare phenomena; pilots do not develop the needed skills for coping with wind shear through normal experience. The typical pilot is likely to be confronted with a life threatening wind shear only once or twice during an entire flying career; hence, it is unlikely that he or she can learn all the important signs of wind shear and maintain a high level of proficiency in the proper control procedures.

On-board computation provides an excellent opportunity to assist the pilot in surviving encounters with severe wind shears, but the logic that must be executed in real time is complex and must have sufficient inputs for framing decisions about appropriate control actions. The computer program(s) and hardware to perform this task must have attributes of expert systems and control systems, they must account for the limitations of aircraft performance, and they must operate in real time. At least as imporant as its technical specifications,

1 Professor.

2 Research Assistant. the on-board system must provide a satisfactory interface with the flight crew, which bears the ultimate responsibility for assuring safety. This means not only that the system must deduce near-optimal strategies and tactics for emergency situations but that it must distinguish between truly hazardous conditions and the more likely alternatives associated with normal aircraft operations.

\section{Background on Wind Shear Encounter}

The microburst phenomenon can be visualized as a vertically descending column of air that spreads out upon hitting the ground $[1,2]$. In a typical scenario, the aircraft first experiences a headwind as it enters the outflow, causing the aircraft to balloon above the flight path if no corrective action is taken (Fig. 1). (This phase may be preceded by an encounter with upflow and tailwinds at the edge of a ring vortex.) Just as the pilot is throttling back to accommodate the headwind, it diminishes and is replaced by a downdraft that is soon followed by a tailwind (and perhaps another ring vortex encounter). The rapid loss of airspeed and the low energy state of the aircraft both contribute to a trajectory undershoot that can lead to ground impact.

A good deal of research has been conducted on the wind shear hazard during the past decade. Much of it deals with the meteorological details of the phenomenon or general treatments of aircraft stability and ad hoc piloting effects [314]. Linear-quadratic regulators with feedforward control that provided remarkably good results in reduced-order linear simulations of wind shear encounter were developed [15]. This work illustrated the potential value of using "energy state", $E$ $=V^{2} / 2 g+h$, either as a feedback variable or as an element of the quadratic cost function.

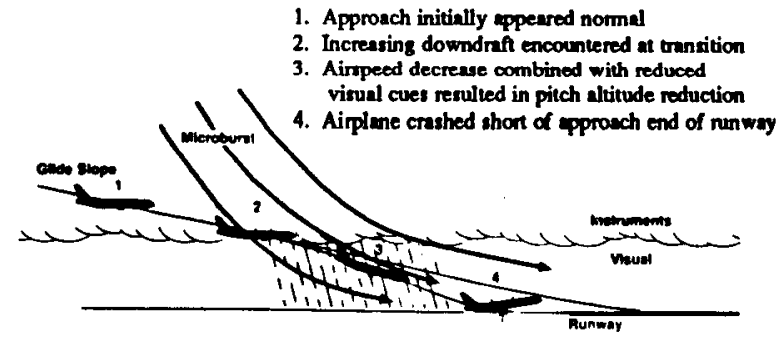

Figure 1. Wind shear encounter during approach (from "Windshear Training Aid," Federal Aviation Administration, Washington, DC, Feb 1987).

Additional applications of the energy state in aircraft guidance and control have been considered [16-22], and a Wind Shear Hazard Index that derives from the combined effects of the vertical shear in headwind and the downdraft magnitude on $d E / d t$ was presented in Ref. 8. Following an extensive classical-control analysis, a feedback controller that dramatically reduces wind shear response by feeding flight path angle and the product of dynamic pressure and angle-of-attack perturbation to elevator and $E$ and $d E / d t$ to throttle was derived 
[23]. This study included realistic delays associated with short period, throttle, and elevator dynamics, which are important in real-world implementation.

Optimal flight paths and guidance strategies also have been suggested [24-29]. Knowing the optimal flight profiles corresponding to various wind fields is vitally important to designing and evaluating on-board systems to protect against wind shear, as survival may well depend on the specific control profile chosen by the pilot. Sub-optimal systems can provide an increased safety margin over conventional cockpit instrumentation, and a number of wind-shear-warning systems are commercially available. Nevertheless, one would like much more than sub-optimal protection through systems that properly anticipate dangerous conditions before they occur and that provide near-optimal guidance in inadvertent encounters. It is in this regard that concepts of machine intelligence may prove useful.

\section{Background on Intelligent Guidance and Control}

The human pilot is a most capable and robust controller, but the complexity, scope, and flight-criticality of making proper decisions and controlling the aircraft during wind shear encounter is an extreme challenge. The nature of the task is not, in any sense, beyond human understanding, but the need for assimilating a multitude of diverse data and taking correct actions may be beyond unaided human capability. Given time and information, an expert crew could arrive at a satisfactory solution; however, it is clear that computers could assess the quantitative aspects of the situation far more quickly than a human can. It would be desirable for a computer to evaluate symbolic, qualitative information as well, although the best way of doing this remains to be found.

A dynamic expert system will form a critical part of an on-board system to counter wind shear effects. Whereas static expert systems may consider fixed vectors of information, many components of the dynamic expert system's information vector are essentially time-varying, increasing the dimensionality of the information. As an example, consider the pattern-recognition problem. In the static case, a fairly straightforward discriminant function could be used to classify information; while in the dynamic case, a discriminant function must be applied to time series of data. In this instance, the typical "IF...THEN...ELSE" paradigm (in its simplest form) is inadequate, and hypothesis testing must be based on dynamic algorithms.

There are two principal approaches to interconnecting machine intelligence and automatic control, based on federated systems and integrated systems. In federated systems, the goal is to provide a symbolic supervisory control for a largescale process control system, as used, for example, in the chemical industry. A LISP processor performing the first class of tasks could be connected to a conventional computer performing the second set of tasks. This decoupling of fundamentally different types of tasks provides good isolation for software development, and it facilitates the manual intervention of a skilled operator, should that be required. However, it is most appropriate for intrinsically slow operations. Integrated systems are Rule-Based Controllers (RBC) that combine symbolic and numeric processing in a highly integrated, parallel environment [30]. The principal characteristics of an integrated system are that numeric processes occur as side effects of forward and/or backward chaining, and the entire system is implemented for real-time execution in an array of similar processors coded with a procedural language.

Three areas appear to be critical to successful real-time implementation of machine intelligence in wind shear encounter. The first is acquisition of sufficient data on which to base a decision and to control the aircraft. The second is the definition of efficient hardware and software structures for a real-time expert system that addresses the wind shear problem. The third is the design of interfaces between the equipment and the pilot that are both complete and reasonable.

A real-time expert system for fault-tolerant control that has many of the features needed for an intelligent anti-windshear system has been developed [30-32]. That system provides a number of important pathfinders for such a system, including implementation of a controller with hierarchical structure, multiple cooperating expen systems in parallel microprocessors, and closure of an intelligent feedback control loop at a high sampling rate. The system structure has been developed using LISP, then translated semi-automatically to Pascal code for real-time operation on three 80286/7 Multibus boards. There are equivalent expert systems in the two languages, both of which access the same database and external simulation

\section{FAA WINDSHEAR TRAINING AID}

The FAA Windshear Training Aid was prepared with the support of the Integrated FAA Wind Shear Program. This two-volume manual was written by a team from the airframe industry that interacted with airlines, government, and academia [33]. Principal results are expressed in a variety of ways for executive review, training classes, and public information. One principal goal is to identify the logical connections between pilot observations and pilot actions when wind shear is encountered. The functions that a jet transport aircraft crew should perform are summarized by a flow chart (Fig. 2).

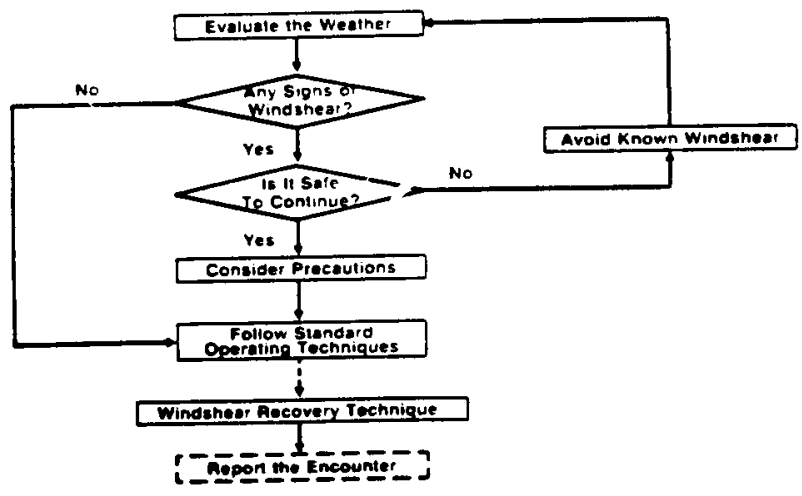

Figure 2. Model of flight crew actions. (from [33])

Flight crews are provided with guidelines that relate observations to wind shear probability along with examples of their use. If the probability of wind shear is LOW, standard procedures are recommended. If the probability is MEDIUM, the crew is instructed to consider precautions, including delay or alteration of terminal operations. If the probability is HIGH, delay or alteration of terminal operations is recommended, with specific actions guided by flight phase. If more than one observation suggests dangerous wind shear, the subjective probabilities should be added, although the guidelines for the risk assessment and the probability addition are imprecise. For example, two LOWs equal a MEDIUM, and either two MEDIUMs or a LOW and a MEDIUM equal a HIGH. There is no guidance regarding spatial or temporal characteristics of the observations; issues of proximity and degree of intensity are left to the pilot's judgment.

Although the strongest suggestion for piloting strategy is "avoid, avoid, avoid," recommended procedures for recovery or abort following wind shear encounter are given as 
functions of flight phase. These strategies are sub-optimal, but they materially enhance the probability of survival, in comparison to standard piloting procedures.

The FAA Windshear Training Aid is a significant achievement in the fight against the hazards of low-altitude wind shear. Nevertheless, it takes a high level of piloting awareness and skill to evaluate the situation and to execute the implied actions correctly and quickly erough to avert catastrophe. To the extent that a computer can be fast and precise, it could assist the flight crew in this dangerous situation.

In seeking to build a computer aid for wind shear avoidance, it is necessary to model the implied logical patterns that the flight crew must use and to quantify subjective rules for computation. Many factors related to situational awareness, limitations to effective action, and efficient decision analysis must be considered, for the computer cannot exert "sound judgment," as suggested in the Training Aid, without having been programmed to do so.

\section{WINDSHEAR SAFETY ADVISOR}

The WindShear Safety Advisor (WSA) is a computer program that uses concepts drawn from the world of artificial intelligence (AI) to assess the wind shear threat and to recommend safe piloting action (Fig. 3). The current version is an

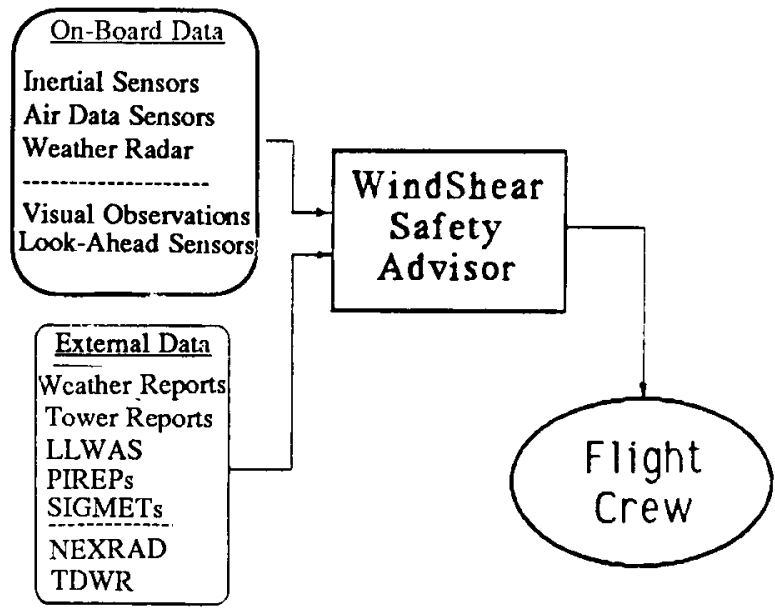

Figure 3. Structure of WindShear Safety Advisor.

interactive but non-real-time program for studying the input information and logic required to emulate and extend the FAA Windshear Training Aid to on-board computer systems. In particular, the WSA implements the stated rules of the Training Aid, and its development is uncovering the unstated (but critical) implications of the manual. The WSA, currently on a Symbolics 3670 LISP machine using the Genera environment, does not address important human factors issues, such as presentation of information to the pilot and requests for pilot input or intervention, which would have little significance in nonreal-time simulation. However, our goal is to identify a program structure that is appropriate for real-time use.

As shown in Fig. 3, the WSA accepts data from sources that are external and internal to the aircraft. External sources include human controllers in an airport control tower, weather reporting systems, terminal doppler weather radar (TDWR), the low-level windshear alen system (LLWAS), and pilot reports (PIREPs). Internal data are transmitted from motion sensors, weather radar, visual observations, and (in the future) "look-ahead" sensors such as infrared, radar, or laser devices.

\section{Functions of the WindShear Safety Advisor}

The WindShear Safety Advisor, in its current state, is principally a model of pilot and crew decision-making and control, as described in the FAA Windshear Training Aid. The four primary functions of the system are: $\begin{array}{ll}\text { MONITORING - } & \begin{array}{l}\text { Observe sensors, receive reports and } \\ \text { flight-plan revisions, alerts, and }\end{array}\end{array}$ warnings

ASSESSMENT - Detect wind shear encounters, determine if there are signs of wind shear, and if it is safe to continue

PLANNING - $\quad$ Recommend actions and precautions to be taken

ACTION - In automatic mode, execute standard, recovery, and go-around procedures; in semi-automatic mode, issue commands to flight directors

These functions are performed as side effects of a goal-directed search for parameter values in a set of rules.

An on-board implementation of the WSA would be a Rule-Based Control (RBC) system having attributes of both expert systems and conventional controllers (Fig. 4). A decision-making process implemented as an expert system may require that certain side tasks be accomplished, such as taking

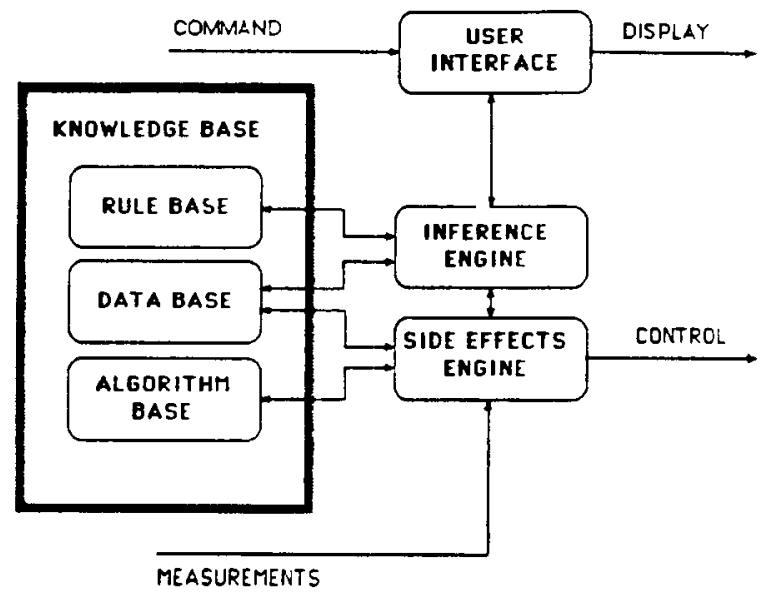

Figure 4. Rule-based system for control.

measurements, making estimates, computing control settings, and transferring commands to control effecters. This procedural, quantitative computation is done in a Side Effects Engine that calls on both the Data Base and an Algorithm Base for its knowledge. (Measurement and control are considered side effects of the request for information and the decisionmaking process.)

Decision and control functions are readily separated in an RBC system, the former calling for symbolic computation, the latter for numeric computation. (In either case, the digital computer simply moves bits around; however, interpretations of the logical operations are different.) Not surprisingly, some computer programming languages are better than others at performing the two types of tasks, so it is most efficient to use different languages for decision and control during the development phase. For example, LISP is a good language for developing logical relationships among strings of symbolic data, while Pascal or FORTRAN is a good language for numerical 
computation. Consequently, LISP is the language of choice for current WSA development. Once decision and control functions have been defined, they must be merged (in some sense) in the RBC system. Development of a real-time version of an RBC system is thus aided by one or more language translators that efficiently transform subsets of the development languages into the final code.

\section{Expert System Techniques and Implementation}

An important feature of the RBC system is its use of a declarative representation of knowledge in a knowledge base. The rules and given facts are structured using frames. The elements of decision making needed for the WSA are illustrated by this simple example (Fig. 5). A parameter is a quantity that
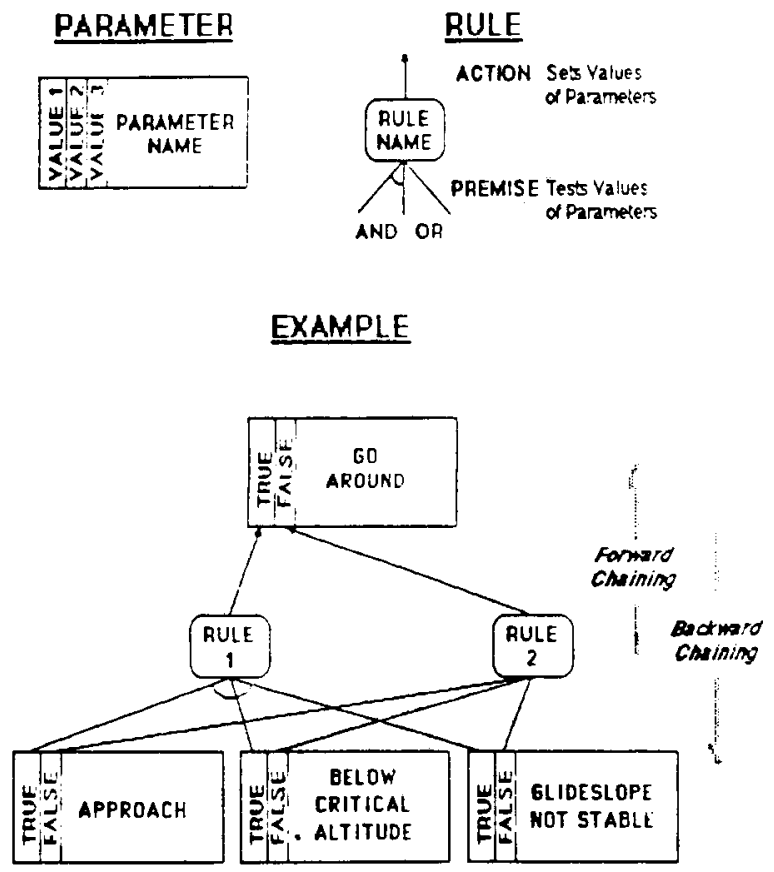

Figure 5. Graphical representation of knowledge.

can have several values as well as an array of complicated properties (not shown). A rule accepts one or more parameters as its premise and performs the action of setting another parameter if the values of its input parameters make the rule true. For the premise to be true, it may be necessary that all multiple parameters take certain values (represented by the arc between connecting lines), or it may be sufficient for any parameter to take a cerain value (represented by no arc between channels into the rule).

The process of applying the knowledge base to given facts is known as inference. Given a base set of facts and rules, all of the facts that follow may be asserted by examining all the rules, in any order, repeatedly, until no new facts can be proven. However, the order in which the rules are tried can make a big difference in the efficiency of this process. With a large number of rules (which is always the case for any substantial amount of exper knowledge), efficient inference in an expert system requires a strategy for deciding the order in which the rules are examined.

Two different types of inference procedures are used in the WindShear Safety Advisor. These procedures can be distinguished by how the expert system obtains facts from the user. With one type of procedure, the user gives a set of facts to the system, and the procedure works forward from these facts, deriving all the possible consequences of them. This type of procedure is called data-driven or forward-chaining inference. With the other type of procedure, the user directs the system to find the truth or falsehood of a goal parameter, and the search procedure works backward from this goal parameter, asking the user to supply values for parameters whose values are not known, relevant to the goal parameter, and cannot be given values by examining rules. This type of procedure is often called goal-directed or backward-chaining inference. The WindShear Safety Advisor uses both types of procedures. Taken together, these procedures are referred to as the inference engine.

To demonstrate the actions of goal-directed inference, consider the following example (Fig. 6). The expert system is

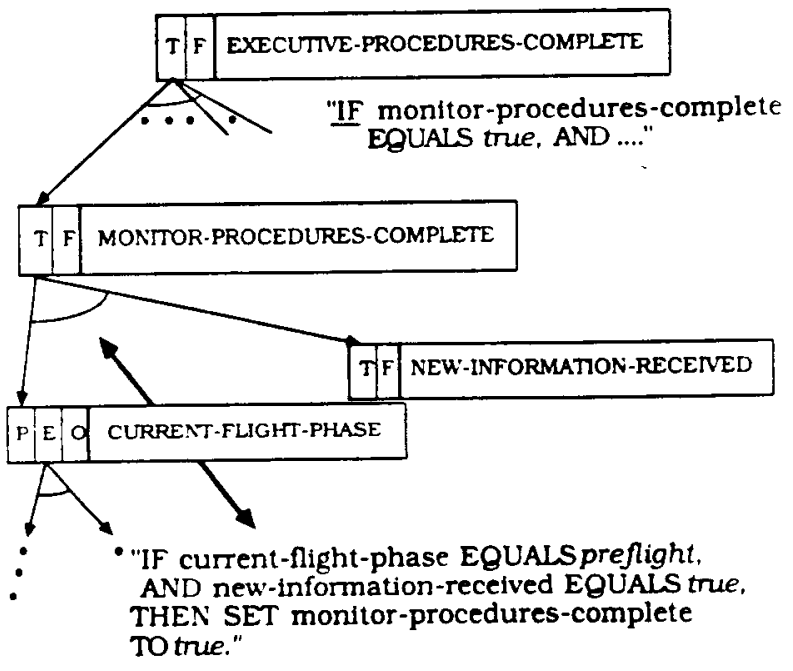

Figure 6. Graphical representation of goal-directed inference.

invoked periodically from a simulation by directing the inference procedure to get a value for the parameter "Executiveprocedures-complete". "Executive-procedures-complete" is set in the EXECUTIVE rule base by the top-most rule in the figure, which considers the truth or falsehood of the expression, "Monitor-procedures-complete". To know this, we must find out what the flight phase is. Turning to the list of parameters, we find a parameter "Current-flight-phase" that is set in the FLIGHT PHASE rule base. The rules within the FLIGHT PHASE rule base are examined next. When the flight phase has been determined, we return to considering the truth or falsehood of the first premise. If, for example, we determined that the flight phase was "Preflight," we would then consider whether or not new information had been received. Once this search is complete, the parameter "Executive-procedures-complete" is set to TRUE, and the toplevel search is finished.

A data frame is a structure that defines the attributes of objects, as well as their linkages to other frames in the knowledge base. We might, for example, describe a hazardous volume of airspace as a sphere of given location and radius (Fig. 7). The sphere frame has two slots: location of the center and radius. The latter is specified by a number, while the former is itself another frame that contains four slots, and so on. Each sphere is denoted by a frame instance, in which actual values appear in the slots. When a specific sphere is defined, it is said to be "instantiated." 


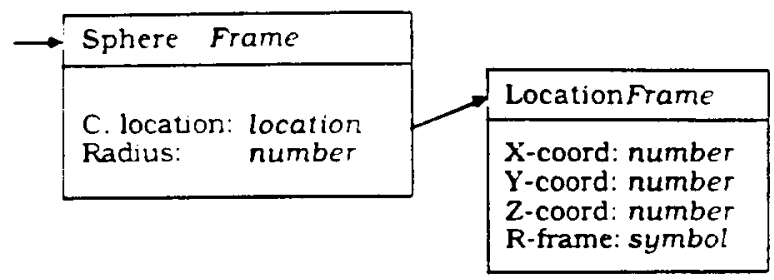

Data stored as frame instances

\begin{tabular}{|c|c|}
\hline Spherei & nstance \\
\hline $\begin{array}{l}\text { X-coord: } \\
\text { Y-coord: } \\
\text { Z-coord: } \\
\text { Frame: } \\
\text { Radius: }\end{array}$ & $\begin{array}{l}0.0 \\
0.0 \\
O .0 \\
R E F \\
3.0\end{array}$ \\
\hline
\end{tabular}

Figure 7. Structuring data with frames.

The WSA contains numerous other frames that describe the aircraft's dynamic state and its so-called "world environment." For example, the flight phase, event, and hazard frames are defined as follows, where terms in italics refer to specific data types:

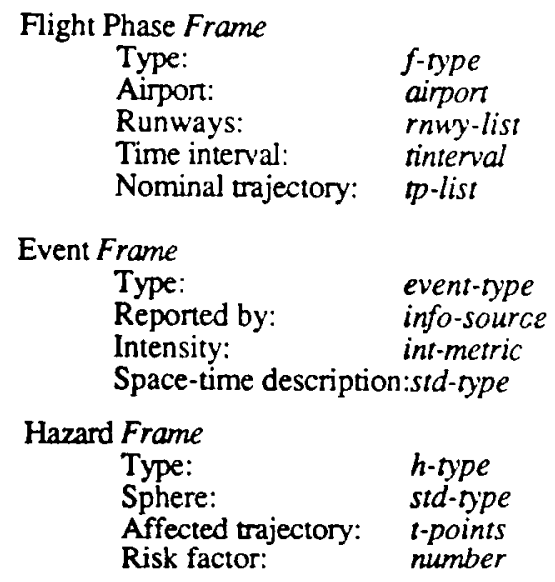

Qualitative decisions must be made on the basis of information contained in these frames, the risk of wind shear encounter providing a good example. An appropriate rule is

IF there is an instance of event type microburst, AND we intersect it in the next flight phase, AND a value for severity is obtained from the microburst-indicator rule base,

THEN a microburst hazard is instantiated, AND the risk factor is increased by the incremental severity value.

The Windshear Training Aid guidelines provide ad hoc way's of determining incremental severity values, but a great deal is left to the implied good judgment of the human decisionmaker. Computer-based decisions require an approach that is at least quasi-quantitative if not rigorously so. For example, the LOW-MEDIUM-HIGH descriptors of wind shear probability can be equated to an ordinal ("1-2-3") scale to implement the above logic. As increasingly numeric information is gathered about risk factors, fractional severity metrics then can be incorporated. Rainshowers on the flight path might reasonably indicate a higher concern than showers near the path but not high enough to increment the risk factor by an integer value.

Another important feature of any expert system is an interface to translate a user's problem into a collection of symbols and to translate the results of inference back into a format the user can understand. The WindShear Safety Advisor Interface, used for logic development and demonstration, is a multiple-window interactive display (Fig. 8). The current version accepts keyboard and mouse input, with the results of inference displayed as text output. Execution of the system consists of an elementary dialogue between the program user and the exper system. As the user answers the system's questions, statements of applicable rules are typed out in one window, while information about parameters is displayed in another. Special messages summarizing the system's advice are printed in a third window.

\begin{tabular}{|c|c|}
\hline \multicolumn{2}{|c|}{ Princeton WindSnear Sofety Advisor interface } \\
\hline \multicolumn{2}{|c|}{ Flight Plon Get Volue of Moke Messoge Presets ….... (Menul Line) } \\
\hline User Interaction Pane & Porameter Informetion Pone \\
\hline $\begin{array}{l}\text { Messages to program } \\
\text { developer } \\
\text { Messages to crew, tower, etc. } \\
\text { Data and commands from } \\
\text { program developer }\end{array}$ & $\begin{array}{l}\text { Parameters that have changed } \\
\text { values } \\
\text { Other parameters of interest }\end{array}$ \\
\hline \multicolumn{2}{|c|}{ Result Monitaring Pere } \\
\hline $\begin{array}{l}\text { Execulive observations } \\
\text { Monitored information } \\
\text { Status assessment }\end{array}$ & $\begin{array}{l}\text { Planning activity } \\
\text { Recommended action } \\
\text { Current airport weather }\end{array}$ \\
\hline
\end{tabular}

Figure 8. Development screen for WindShear Safety Advisor.

\section{CONCLUSIONS}

The WindShear Safety Advisor program implements the stated decision-making logic of the FAA Windshear Training Aid, as well as a set of unstated implications that are necessary for practical application. The WSA expert system contains over 200 rules that set over 80 parameters for terminal operations of jet transport aircraft. Future modifications will account for spatial and temporal variations of the aircraft and its meteorological environment, as well as for interfaces with the air traffic control system. The WindShear Safety Advisor sets the stage for cockpit simulation of logic for wind shear avoidance, which, in turn, will lead to practical systems for operational aircraft.

\section{ACKNOWLEDGMENT}

This research has been sponsored by the NASA Langley Research Center under Grant No. NAG-1-834.

\section{REFERENCES}

1. Townsend, J., committee chairman, "Low-Altitude Wind Shear and Its Hazard to Aviation," National Academy Press, Washington, 1983.

2. Stengel, R., "Solving the Pilot's Wind-Shear Problem," Aerospace America, Vol. 23, No. 3, Mar 1985, pp. 82-85. 
3. Fujita, T., "Downbursts and Microbursts - An Aviation Hazard," Proceedings of the 19th Conference on Radar Meteorology, Miami Beach, 1980, pp. 94-101.

4. McCarthy, J., et al, "The Joint Airport Weather Studies (JAWS) Project," Bulletin of the American Meteorological Society, Jan 1982, pp. 15-22.

5. Bray, R., "A Method for Three-Dimensional Modeling of Wind-Shear Environments for Flight Simulator Applications," NASA TM-85969, July 1984.

6. Zhu, S., and Etkin, B., "Fluid-Dynamic Model of a Downburst," UTIAS Report No. 271, Apr 1983.

7. Bach, R., Jr., and Wingrove, R., "The Analysis of Airline Flight Records for Winds and Performance with Application to the Delta 191 Accident," AIAA Paper No. 86-2227-CP, Aug 1986.

8. Bowles, R., "Fundamental Concepts for Wind Shear Detection, Warning and Flight Guidance," presentation at Airborne Wind Shear Systems Manufacturers Review," Hampton, Oct 1986.

9. Sherman, W., "A Theoretical Analysis of Airplane Longitudinal Stability and Control as Affected by Wind Shear," NASA TN D-8496, July 1977.

10. Gera, J., "The Influence of Vertical Wind Gradients on the Longitudinal Motions of Airplanes," NASA TN D-6430, Sept 1971 .

11. Frost, W., "Flight in Low-Level Wind Shear," NASA CR-3678, Mar 1983.

12. Lehman, J., et al, "Simulation and Analysis of Wind Shear Hazard," FAA-RD-78-7, Dec 1977.

13. Urnes, J., et al, "H-Dot Automatic Carrier Landing System for Approach Control in Turbulence," ALAA Journal of Guidance and Control, Mar-Apr 1981, pp. 177-183.

14. Foy, W., "Airbomè Aids for Coping with Low-Level Wind Shear," FAA-RD-117, July 1979.

15. Rynaski, E., and Govindaraj, K., "Control Concepts for the Alleviation of Windshears and Gusts," NASA CR166022, July 1982.

16. König, R., et al, "Procedures to Improve Flight Safety in Wind Shear Conditions," Proceedings of the 12th Congress of the International Council of the Aeronautical Sciences, ICAS80-22.3, Munich, Oct 1980, pp. 744-757.

17. Lambregts, A., "Vertical Flight Path and Speed Control Autopilot Design Using Total Energy Principles," ALAA Paper No. 83-2239-CP, Aug 1983.

18. Belcastro, C., and Ostroff, A., "Total-Energy-Rate Feedback for Automatic Glide-Slope Tracking During Wind-Shear Penetration," NASA TP-2412, May 1985.

19. Ostroff, A., et al, "Evaluation of a Total-Energy-Rate Sensor on a Transport Airplane," NASA TP-2212, Nov 1983.

20. Jorck, H., "Design of Wind Shear Filters," NASA TM77460 , Nov 1984.

21. Broussard, J., "Extensions to PIFCGT: Multirate Output Feedback and Optimal Disturbance Suppression," NASA CR3968, Mar 1986.
22. Hahn, K., "Take-Off and Landing in a Downburst", Proceedings of the 15th Congress of the International Council of the Aeronautical Sciences, ICAS-86-5.6.2, Sept 1986, pp. 860-869.

23. Psiaki, M., and Stengel, R., "Analysis of Aircraft Control Strategies for Microburst Encounter," ALAA Journal of Guidance, Control, and Dynamics, Vol. 8, No. 5, Sept-Oct 1985 , pp. 553-559.

24. Miele, A., Wang. T., and Melvin, W., "Optimal Take-Off Trajectories in the Presence of Windshear," Journal of Optimization Theory and Application, Apr 1986, pp. 1-45.

25. Psiaki, M., and Stengel, R., "Optimal Flight Paths Through Microburst Wind Profiles," AIAA Journal of Aircraft, Aug 1986, pp. 629-635.

26. Psiaki, M., "Control of Flight Through Microburst Wind Shear Using Deterministic Trajectory Optimization," Ph.D. Thesis, Princeton University, 1987.

27. Miele, A., Wang, T., and Melvin, W., "Optimization and Gamma/Theta Guidance of Flight Trajectories in a Windshear," Proceedings of the 15th Congress of the International Council of the Aeronautical Sciences, ICAS-86-5.6.4, Sept 1986, pp. 878-899.

28. Stengel, R., "Optimal Control Laws for Microburst Encounter," Proceedings of the 15th Congress of the International Council of the Aeronautical Sciences, ICAS-86-5.6.3, Sept 1986, pp. 870-877.

29. Psiaki, M., and Stengel, R. "Performance Limits for Optimal Microburst Encounter, Proceedings of the 1988 AlAA Atmospheric Flight Mechanics Conference, Minneapolis, Aug 1988, pp. 358-370.

30. Handelman, D., and Stengel, R., "An Architecture for Rule-Based Control," Proceedings of the 1987 American Control Conference, Minneapolis, June 1987, pp. 1636-1642.

31. Handelman, D., and Stengel, R., "Combining Quantitative and Qualitative Reasoning in Aircraft Failure Diagnosis," Proceedings of the 1985 AlAA Guidance, Navigation, and Control Conference, AlAA Paper No. 85-1905CP, Aug 1985.

32. Handelman, D., and Stengel, R., "A Theory for FaultTolerant Control Combining Expert System and Analytical Redundancy Concepts, Proceedings of the 1986 AIAA Guidance, Navigation, and Control Conference, AIAA Paper No. 86-2092CP, Aug 1986.

33. -. , "Windshear Training Aid," Federal Aviation Administration, Washington, DC, Feb 1987. 